\section{Secure and private smart grid: \\ the SPEAR project}

Panagiotis Radoglou-Grammatikis and

Panagiotis Sarigiannidis

\section{The digital world we currently} enjoy with intelligent environments and smart

devices has not been

accomplished without difficulty. Intelligent objects make our lives comfortable, more straightforward and automated. However, this digital reality comes with severe cybersecurity and privacy issues due to the vulnerabilities of smart and legacy technologies.

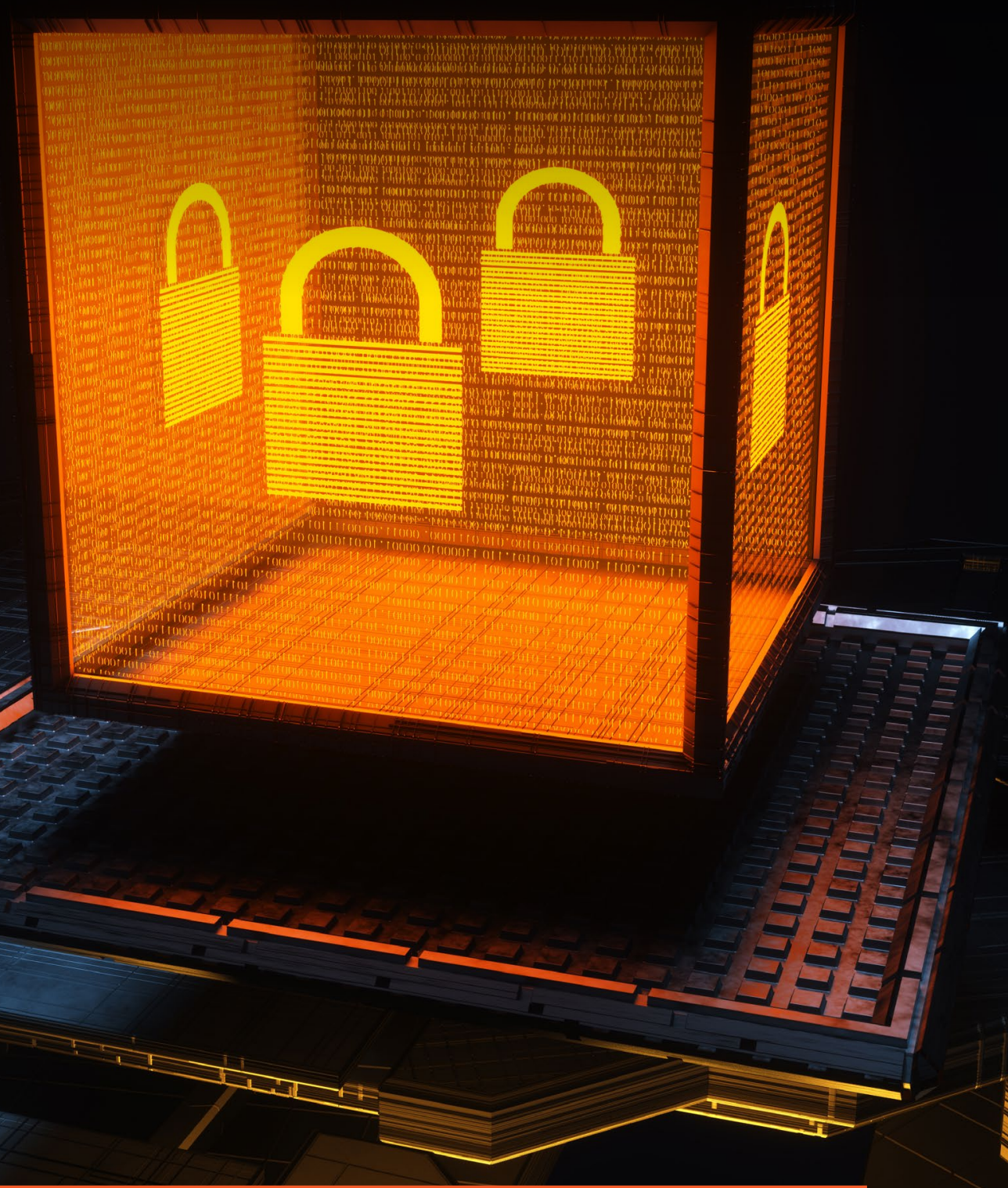

Cyberattacks are socially or politically motivated malicious actions carried out primarily via the weaknesses of the typical internet model. A decade ago, the cyberdefensive mechanisms focused mainly on mitigating and preventing cyberattack targeting websites and web applications Now, given the digital era of the industris internet of things (IloT), a cyberattack against a critical infrastructure (CI) could significantly affect human lives.

Since our society is explicitly dependent on Cls, the cybersecurity mechanisms should be adapted and optimised appropriately to meet the security requirements of $C$ taking into account their sensitive neture takng the account their sensitive nature and pre conting intrusens carr. Detectise hishy-skiled, persistent and coording a houp che of cy The ckers challenging task. The smart gid (SC) is one of the most valies cyber activities can lead to disastrous fatal accidents (Radoglou-Grammatikis \& Sarigiannidis, 2019).

In particular, the SG is considered as th next-generation electrical grid promisin multiple benefits and services, such as selfhealing, improved resilience and pervasive control. However, since this paradigm adopted globally among various energy stakeholders, more and more intruders try to violate the security of the SC assets. In parallel, the SG incorporate legacy assets, such as supervisory contro and data acquisition (SCADA)/industria control systems (ICS), that are vulnerab to a plethora of unauthorised activities due to the necessary presence of insecure communication protocols (Gunduz \& Das, 2020). Characteristic examples are Modbus and IEC 60870-5-104. According to the European Network and Information Security Agency (ENISA): "A cybersecurity incident against electrical power and energy systems (EPES) is considered as any security event, which aims to affect the essential cybersecurity principles related to the involved assets originating either from the electrical ensinecing sector or the information and conng sector technology (ICT) area." Consequently, it is evident that the SG evolution generates enormous cybersecurity and privacy challenges.

Currently, the cybersecurity incidents against Cls have been seriously intensified. One example is the cyberattack on a power outage for over 225,000 peop Other advanced persistent threats (APTs) are DrenFly, Dugu, Flame Sturnet are Dragonfy, Dugu, Flame, Stuxnet, Throv and Dreser consider the unique shecters ponsider the unique charcterstics and pecurther of the mo not consder Eurthermore, they do not consider the capacity of advanced visualisation methods capable of recognising anomalous events and situations. Moreover, the forensic mechanisms are limited only to monitoring and logging (Gunduz \& Das, 2020) activities without extracting clear evidence that can be used in court. Therefore, stealthy attacks can evade the typical countermeasures. Hence, the following challenges are identified: (a) enhancing the EPES/SG resiliency and reliability; (b) detecting and mitigating timely and accurately EPES/SG cyberattacks; (c) addressing multi-step attack scenarios, such as APTs; (d) enhancing digital forensic solution taking into account privacy concerns; (e) optimising the trust among the EPES/SG assets; and (f) empowering EU-wide consensus.

\section{SPEAR objectives}

SPEAR (Secure and PrivatE smArt gR is a Research and Inovation Action (RAA) project funded by the Horizon 2020 framework programme of the European Union, aiming to provide an integrated solution capable of: (a) detecting timely potential cyberattacks/anomalies (e.g. disturbances) against the SG environments; (b) ensuring the presence of sufficient forensic mechanisms collecting the necessary attack traces and preparing the appropriate legal processes; (c) providing an anonymous repository of cybersecurity incidents for the energy sector; and introducing a cyber hysene framewor, evaluting the cybersecuity reathes level of the EPES personnel and providing web-based training environment, which will continually inform and educate the EPES personnel about the best practices in the cybersecurity domain. SPEAR focuses on the following primary objectives.

Objective \#1: define the SPEAR system architecture, the security components and the privacy framework for situational awareness provisioning in

relation to cybersecurity threats

The first objective is related to defining the architecture of the SPEAR solution, which will constitute an integrated platform, comprising: (a) detection and correlation components; (b) a forensic repository; (c) deception components; (d) an anony To this a cyber hygiene framework. To this end, the ARCADE framework (Radoglou-Gramatiks, et al., 2020) will be adopted, tientryling the functional and mon-functional requirements, the data model, the various components and their terfaces.

Objective \#2: build attack detection mechanisms and promote resilience operations in SG

A significant aspect of SPEAR is to accurately detect the various cyberattacks and anomalies against the EPES assets n a timely manner. To this end, machine earning (ML) and deep learning (DL) models have been implemented, detecting plethora of cyberattacks against a protocols, such as Modbus, Distributed Network Protocol 3 (DNP3), IEC 60870-5104, IEC 61850/Manufacturing Message pecification (MMS) Message Queuing Telemetry Transport (MQTT) BACnet, letwork Time Protocol(NTP) Secure Shell SSH) RADIUS and (Nypert, Secure Shel Proter (HTP), The Vensfer Prection cocton into analytics component (BDAC) 
Moreover, SPEAR includes a visual(VIDS) capable of distinguishing
electrical disturbances and other kinds electrical disturbances and other kinds
of anomalies, using advanced visua analytics with operational data (i.e. time series electricity measurements).

Objective \#3: increase situationa awareness in SG networks

After the detection processes, SPEAR aims to enhance situational awareness utilising correlation mechanisms. To th end, based on the various security events desSIM, the GRC, VIDS and Alienaut OsSIM, the Gid Trusted Module (GTM) re-calculates the reputation score of each EPES assets, utilising fuzzy logic rules. The reputation score represents the trust leve of each EPES asset within a sub-network.

Objective \#4: create and

maintain an anonymous

repository of SG incidents

SPEAR also intends to contribute to global situational awareness by creating an maintaining a repository of SG incidents. The rationale behind the creation of and exchange critical information abou cyberattack incidents in SG environments across Europe. To this end, the SPEAR Repository of Incidents (SPEAR RI) wil be implemented and compliant with the best practices designed by relevant organisations, such as the European Energy - Information Sharing \& Analysis Centre (EE-ISAC) and the European Smart Metering Industry Group (ESMIG). SPEAR of the EPES organisations related to cybersecurity incidents. Simultaneously, maintains the technical

Objective \#5: provide smart network forensics subject to data protection and privacy

SPEAR intends to provide a forensic readiness framework (FRF), which wil based intrusion detection system this repository is to broadcast, inform $\mathrm{Rl}$ incorporates appropriate anonymisation

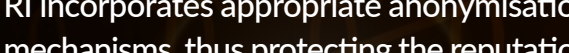
mechanisms, thus protecting the reputation guarantee the digital forensic actions and collection of the evidence, taking into account the privacy of the involved mescar methodology will be followed-obtain (n) analyse and report. Moreover, a data privacy impact assessment (DPIA) the data flows related to the SPEAR components. Finally, in the context of the SPEAR FRF, a forensic repository (FR) will be developed, including visualisation capabilities, thus facilitating the work

\section{OSCAR}

Obtain information

Strategise

Collect evidence

Analyse

Report

Objective \#6: empower EU-wid consensus of cybersecurity in SG systems

Based on the guidelines of NIST SP 800 53 and NIST SP 800-82, there is no current range of capabilities required for smart devices and meters. A risk assessment is needed in all layers of security (physical, smart metering, networking and to significant threats. In the context the SPEAR architecture, authorities and energ un arites can any informetion about their vulnerabilities without exposine critical seosapical cent or techical detais. SPEAR RI will bring orether of trust where the varous a network of trust whe the various entites can cybersecurity incidents.

Objective \#7: validate the

SPEAR architecture capabilities in proof-of-concept use cases

The SPEAR consortium has selected four the forensic investigator. high-impact use cases to validate the SPEAR architecture namely the:

hydropower plant

- substation

combined use case

smart house use case.

Each use case is characterised by different needs and security requirements that are met by SPEAR. Thus, SPEAR can satisfy various EPES cases and stakeholders in detecting, correlating, and mitigating cyberattacks and anomalies.

Objective \#8: design an

innovative business model and

conduct a techno-economic

analysis to strengthen the

role of European SG and the

cybersecurity industry in the global market

One of the main goals of SPEAR is to trengthen the role of the European SC security domain in the global market. Union (EU) SG security industry with the necessary innovation capacity to increase its leading role in the world business field. The main rationale behind this objective is to seek exploitetion orturites of the SPEAR results by pontifying potential SPEAR resuls by cluding a revenue prediction.

\section{SPEAR architecture}

As illustrated by Figure 1, the architecture of SPEAR consists of three main components: (a) SPEAR Security formation and Event Management system (SIEM); (b) SPEAR FRF; and (c) SPEAR RI.

SPEAR SIEM is the flagship of SPEAR, integrating the intrusion/anomaly detection and correlation mechanisms (Radoglou-Grammatikis, et al., 2021). First, SPEAR SIEM uses AlienVault OSSIM with respect to its sisnature/specifcetionthed intrusion detection specificationOSSEC and Surict). SPR stems (i.e. deployed throughout the EPES subnets, SPEAR aims to provide the European collecting the network traffic and operational data.

Next, the Data Acquisition Processing an Storage (DAPS) receives and normalises the data from the various SPEAR by BDAC and VIDS in order to detect potential cyberattacks and anomalies. BDAC applies ML/DL detection mode to recognise security violations against
EPES industrial protocols, while VIDS uses dimensionality reduction technique to identify anomalies concerning the operational data.

GTM then receives the security events generated by BDAC, VIDS and AlienVaut OSSIM and re-calculates the reputation value of each EPES asset. Finally, the message bus represents an asynchronous communication system, which facilitates communicationamong the aforementioned SPEAR SIEM components.

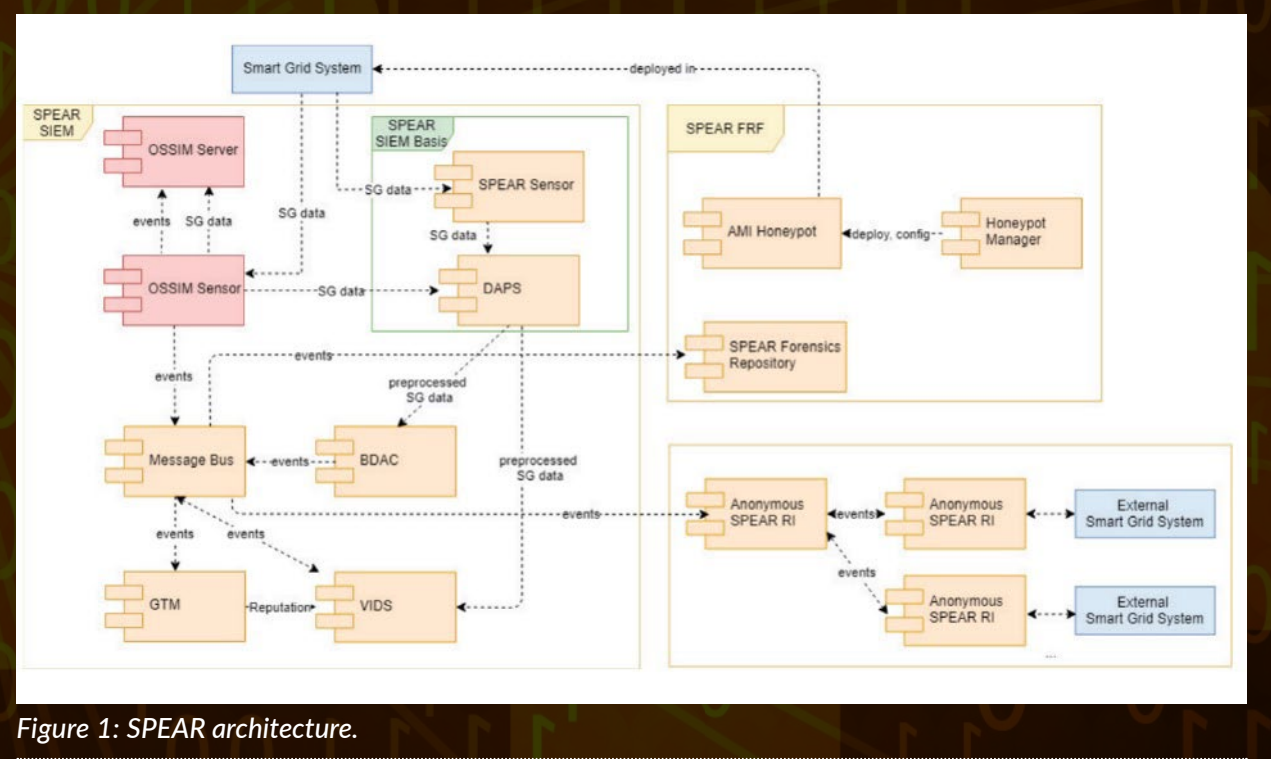

\section{References}

Gunduz, M. Z. \& Das, R., (2020) 'Cyber-security on smart grid: Threats and potential solutions', Computer
Networks, p. 107094.

Radoglou-Grammatikis, P. \& Sarigiannidis, P. (2019) 'Securing the smart grid: A comprehensive compilation
of intrusion detection and prevention systems', IEEE Access, pp. 46595-46620. Radoglou-Grammatikis, P., Iturbe, E., Rios, E., Sarigiannidis, A., Nikolis, O., loannidis, D. Machamint, V., Tziffes,
M., Giannakoullias, A., Angelopoulos, M., Papadopoulos, A. and Ramos, F. (2020) Secure and Private Smart Grid: The SPEAR Architecture. s.l., s.

Radoglou-Grammatikis, P., Sarigiannidis, P., Iturbe, E., Rios, E., Martinez, S., Sarigiannidis, A., Eftathopoulos,
G., Spyridis, Y, Sesis, A., Vakakis, N., Tzovaras, D., Kafetzzakis, E., Giannoulakis, I., Tzifas, M., Giannakoulias,

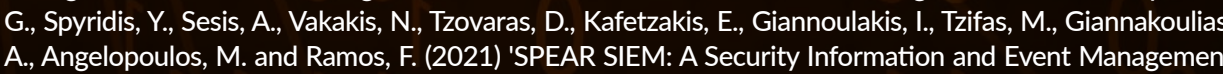
system for the Smart Grid', Computer Networks, p. 10800

On the other side, SPEAR FRF includes three main components: (a) honeypots; (b) repository (FR). Honeypots are intentional security holes that aim to trap potential the honeypots are deployed through the the the benefits and the costs of the defender and the attacker side. The SPEAR FR collects the necessary forensic information related the various security violations, including the honeypots logs and security events RI receives, anonymises and distributes the security events generated by SPEAR SIEM Of various EPES organisations. To this end, Sharing Platform (MISP) platform. It is SPEAR into an integration environment with a common dashboard. sensors. The normalised data is use

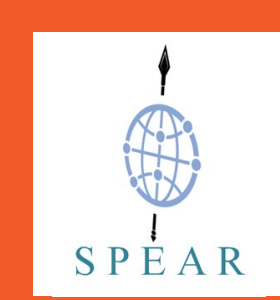

Adobe Stock $\Theta$ Irina

www.europeandissemination.eu
PROJECT SUMMARY

The rapid evolution of the smart technologies has digitised the electrical grid into a new multiple benefits, this reality raises severe cybersecurity concerns. SPEAR provides a
global cybersecurity solution, which (a) detects global cybersecurity solution, which (a) detects
SG-related cyberthreats, (b) provides an SG-related cyberthreats, (b) provides an
advanced forensic readiness framework; and advanced forensic readiness framework; and
(c) introduces an SG cyber hygiene framework

\section{PROJECT LEAD}

SPEAR provides a global cybersecurity solution, which (a) detects SG-related cyberthreats, (b) provides an advanced forensic readiness framework; and (c) introduces an SG cyber
hygiene framework. The main achievements of Sygiene framework. The main achievements of Management System, (b) SPEAR Repository of
Incidents (SPEAR RI), (c) RTU Honeypot; and (d)
NeuralPot. PROJECT PARTNERS

University of Western Macedonia (UOWM)

European Dynamics Luxembourg SA (ED)

Fundacion Tecnalia Research \& Innovation (TEC

Enel lberia SRL (ENEL)

Public Power Corporation S.A. (PPC)

Eight Bells Ltd (8BL)

Incites Consulting SA (INC)

G.E. Pukhov Institute for Modeling in Energy

Engineering of the National $A$ cad
Sciences of Ukraine (PIMEE)

Gottfried Wilhelm Leibniz Universitaet

Hannover (LUH)

Sidroco Holdings Ltd (SID)

O Infinity Ltd (OINF)

Technical University of Soffia (TUC)

MVETS Lenishta OOD (VETS)

Schneider Electric Espana SA (SCHN)

CONTACT DETAILS

Panagiotis Sarigiannid

Karamanli \& Ligeris, Kozani, Greece 50100

(1) https://ithaca.ece.uowm.gr

in /panagiotis-sarigiannidis-7636901a

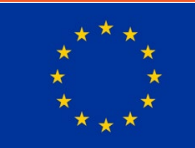

FUNDING

This project has received funding from the European
Union's Horizon 2020 research and innovation Union's Horizon 2020 research and innovat
programme under grant agreement №. 787011. 